\title{
Assessment of laparoscopic suturing skills of urology residents: A Pan-European study
}

Kroeze SG, Mayer EK, Chopra S, Aggarwal R, Darzi A, Patel A

Department of Urology, St Mary's Campus, Imperial College Healthcare NHS Trust, London, UK

Eur Urol. 2009; 56: 865-73

Background: It has been acknowledged that standardised training programmes are needed to improve laparoscopic training of urologic trainees. Previous studies have suggested that simulator-based laparoscopic training can improve performance during real laparoscopic procedures.

Objective: To determine if there are performance differences for the completion of a simulated laparoscopic suturing task among urology residents based on their postgraduate year of training (PGY).

Design, Setting, and Participants: Using a validated scoring checklist, two independent observers objectively scored the completion of a standardised laparoscopic suturing task in a bench-top laparoscopic box trainer. PGY and previous exposure to laparoscopic surgery and laparoscopic simulated training was obtained from selfadministered questionnaires. Data acquisition was undertaken at the European Urological Residents Education Programme (EUREP) 2007, run by the European School of Urology, and included a pan-European cohort of 201 urology residents.

Measurements: Reliability among those rating the suturing tasks was excellent (Cronbach's alpha=0.83). Each resident was scored for the suturing task. Residents were categorised into three groups based on their PGY status (junior [ $n=8]$; intermediate $[n=37]$; senior $[n=156]$ ). The Kruskal-Wallis test was used to measure trend across the PGY; the Mann-Whitney U test was used to determine variation among categorised PGY groups.

Results and Limitations: Laparoscopic suturing skill was significantly different across PGY levels ( $\mathrm{p}=0.032)$, and between junior residents and both intermediate and senior residents $(\mathrm{p}=0.008$ and $\mathrm{p}=0.012$, respectively). There was no significant difference between intermediate and senior residents $(\mathrm{p}=0.697)$. Only $12 \%$ of participants rated their existing volume of laparoscopic operative cases as sufficient, while $55 \%$ of participants had no regular opportunities, and $32 \%$ of participants had not performed laparoscopic procedures as primary surgeon. Most residents (96\%) reported the use of laparoscopic simulators to be beneficial in training, although current European training programmes appear to provide $<50 \%$ of residents with the opportunity to train with them.

Conclusions: A discernable relationship existed between the score obtained for a laparoscopic suturing task and year of resident training. Modular simulator training as part of a formal training programme may help to overcome some of the shortfall in residents' exposure to laparoscopic procedures as primary surgeon.

\section{Editorial Comment}

The optimal educative tools and transfer of knowledge in laparoscopic urological procedures have not been established yet. The authors have evaluated the acquisition of laparoscopic suturing techniques performed by different levels of European residents (201 residents) in different programs. The evaluation and data acquirement was performed by the European Urological Residents Education Program and the residents had to answer a survey. The conclusion of this study revealed that laparoscopic suturing skill was significantly different between junior residents and both intermediate and senior residents but there was no significant difference between intermediate and senior resident. Only $12 \%$ of trainees felt that they had enough laparoscopic operative experience, while $55 \%$ of participants had no regular opportunities, and $32 \%$ of participants had not performed laparoscopic procedures as primary surgeon. Most residents (96\%) reported the use of laparoscopic simulators to be beneficial in training, although current European training programs appear to provide less than $50 \%$ of residents with the opportunity to train with them. In conclusion, there is a clear direct relationship between the skill level of laparoscopic suturing task and year of resident training and the residents perceive that laparoscopic 
simulators may help formal laparoscopic training to overcome the lack of exposure to laparoscopic procedures as primary surgeons.

Dr. Fernando J. Kim

Chief of Urology, Denver Health Med. Ctr. Associate Professor, Univ. Colorado Health Sci. Ctr. Director of Minimally Invasive Urol. Oncology, UCHSC

Denver, Colorado, USA

E-mail:fernando.kim@dhha.org

\section{IMAGING}

\section{T1 hyperintense renal lesions: characterization with diffusion-weighted MR imaging versus contrast-enhanced MR imaging}

Kim S, Jain M, Harris AB, Lee VS, Babb JS, Sigmund EE, Rueff LE, Taouli B

Department of Radiology, New York University Medical Center, New York, NY, USA

Radiology. 2009; 251: 796-807

Purpose: To compare the performance of apparent diffusion coefficient (ADC) measurement obtained with diffusion-weighted (DW) magnetic resonance (MR) imaging in the characterization of non-fat-containing T1 hyperintense renal lesions with that of contrast material-enhanced MR imaging, with histopathologic analysis and follow-up imaging as the reference standards.

Materials and Methods: Institutional review board approval was obtained for this HIPAA-compliant retrospective study, and the informed consent requirement was waived. Two independent observers retrospectively assessed MR images obtained in 41 patients with non-fat-containing T1 hyperintense renal lesions. The MR examination included acquisition of DW and contrast-enhanced T1-weighted images. For each index lesion, the observers assessed the (a) mean (+/- standard deviation) of ADC, (b) enhancement ratio, and (c) subtracted images for the presence of enhancement (confidence score, 1-5). Histopathologic analysis of renal cell carcinomas (RCCs) and follow-up imaging for benign lesions were the reference standards. ADCs of benign lesions and RCCs were compared. Receiver operating characteristic (ROC) curve analysis was performed to assess the accuracy of DW imaging, enhancement ratio, and subtraction for the diagnosis of RCC.

Results: A total of 64 lesions (mean diameter, $3.9 \mathrm{~cm}$ ), including 38 benign T1 hyperintense cysts and $26 \mathrm{RCCs}$, were assessed. Mean ADCs of RCCs were significantly lower than those of benign cysts $([1.75+/-0.57] \mathrm{x}$ $10(-3) \mathrm{mm}(2) / \mathrm{sec}$ vs $[2.50+/-0.53]$ x $10(-3) \mathrm{mm}(2) / \mathrm{sec}, \mathrm{P}<.0001)$. ADCs of solid and cystic portions of complex cystic RCCs were significantly different $([1.37+/-0.55]$ x $10(-3) \mathrm{mm}(2) / \mathrm{sec}$ vs $[2.45+/-0.63]$ x $10(-3)$ $\mathrm{mm}(2) / \mathrm{sec}, \mathrm{P}<.0001)$. When data from both observers were pooled, area under the ROC curve, sensitivity, and specificity were $0.846,71 \%$, and $91 \%$, respectively, for DW imaging; $0.865,65 \%$, and $96 \%$, respectively, for enhancement ratio (at the excretory phase); and $0.861,83 \%$, and $89 \%$, respectively, for subtraction $(\mathrm{P}=.48$ and $\mathrm{P}=.85$, respectively). The combination of DW imaging and subtraction resulted in area under the ROC curve, sensitivity, and specificity of $0.893,87 \%$, and $92 \%$, respectively, with significantly improved reader confidence compared with subtraction alone $(\mathrm{P}=.041)$.

Conclusion: The performance of DW imaging was equivalent to that of enhancement ratio in the characterization of T1 hyperintense renal lesions, with both methods having lower sensitivity than image subtraction without reaching significance. 